\title{
Hyperammonaemic encephalopathy after a subureteric injection for vesicoureteric reflux
}

\author{
S M Zuberi, J B P Stephenson, A F Azmy, P H Robinson, R C McWilliam
}

\begin{abstract}
A 6 year old boy developed hyperammonaemic encephalopathy following a subureteric injection for treatment of vesicoureteric reflux. The hyperammonaemia may be explained by a postoperative urinary tract infection with a urea splitting organism, leading to raised urine ammonia that was absorbed easily across a dilated urinary tract. Agitation and alteration in consciousness level following a urological procedure, in a child with a dilated urinary tract, may be signs of a treatable hyperammonaemic encephalopathy.

(Arch Dis Child 1998;79:363-364)
\end{abstract}

Keywords: hyperammonaemia; encephalopathy; subureteric injection; urea splitting organism

In our hospital approximately 70 children have endoscopic treatment for vesicoureteric reflux every year. A synthetic paste, Macroplastique (polydimethylthiloxane; Uroplasty bv, Maastricht, the Netherlands), is injected under the ureter as it passes through the bladder wall. We report a child who developed hyperammonaemic encephalopathy and subsequent profound brain injury within days of this procedure.

\section{Case report}

A 6 year old boy underwent subureteric injection, after imaging studies had shown bilateral renal scarring, hydronephrosis, and hydroureter all suggestive of grade IV reflux uropathy. He was discharged the same day on oral amoxycillin-clavulanic acid. Four days later he returned to hospital complaining of abdominal pain and passing small amounts of cloudy, blood stained urine. The bladder was drained of $400 \mathrm{ml}$ of urine. He developed increasing agitation and reduced urine output 36 hours after admission. Over the next 24 hours he became increasingly drowsy, showing abnormal episodic flexion of the upper limbs. Brain computed tomography (CT) was reported as normal. He continued to deteriorate, and within eight hours he was comatose with decerebrate posturing and dilated, poorly responsive pupils. He was treated initially with intravenous mannitol and intermittent positive pressure ventilation.

Plasma ammonia was $320 \mu \mathrm{mol} / 1$ (normal $<40$ ); glucose, liver function tests, and coagulation studies were normal. Ultrasound showed debris within dilated upper urinary tracts and bilateral nephrostomies were placed. Urine $\mathrm{pH}$ was 9.0 (normal range 4.5-8.0) and it contained pus cells, blood, and protein, but no

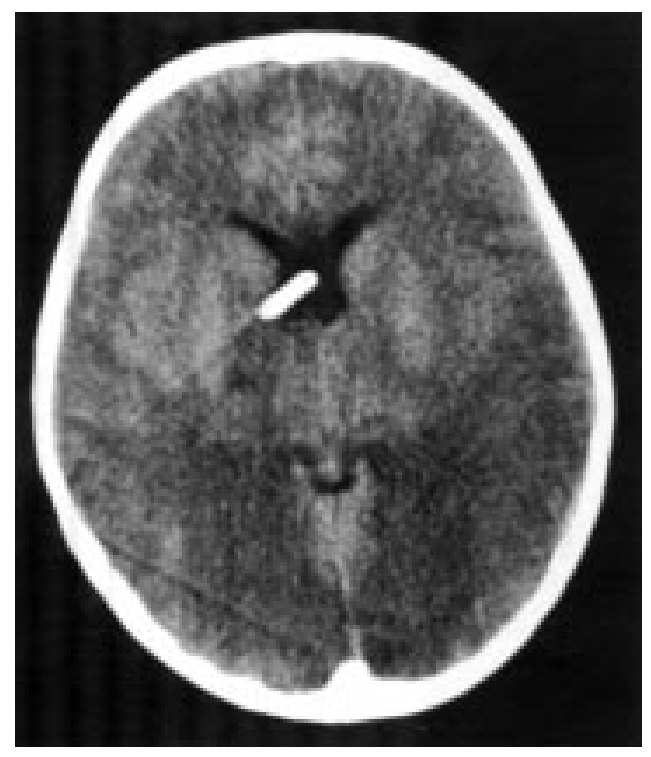

Figure 1 CT six days after transtentorial herniation. There is extensive ischaemia in the temporal, low parietal, and occipital lobes.

organisms were seen or isolated on culture. Urine ammonia was $98 \mathrm{mmol} / 1$ (normal range 0.52-1.08 mmol $/ \mathrm{kg} / 24$ hours). Intravenous ceftazidime was started.

Plasma ammonia fell to $190 \mu \mathrm{mol} / \mathrm{l}$ over three hours following urinary drainage. Intravenous arginine, sodium benzoate, and sodium phenylbutyrate were given along with carnitine. Within two hours, plasma ammonia had fallen to $72 \mu \mathrm{mol} / 1$. Plasma urea $(18.9 \mathrm{mmol} / \mathrm{l})$ and creatinine $(144 \mu \mathrm{mol} / \mathrm{l})$ were raised but returned to normal within 48 hours. Repeat CT showed cerebral oedema.

Plasma and urinary amino acids, urinary organic acids, orotic acid, and carnitine were normal. Plasma glutamine was in the high normal range $(957 \mu \mathrm{mol} / \mathrm{l})$ and cerebrospinal fluid glutamine $(1345 \mu \mathrm{mol} / \mathrm{l})$ and glutamate $(26.8 \mu \mathrm{mol} / \mathrm{l})$ were raised reflecting hyperammonaemia.

He was ventilated for 12 days and subsequent CT showed evolution of a bilateral posterior cerebral artery infarction (fig 1). Ten months later he is registered blind and has an asymmetric spastic tetraparesis, with gradually improving speech and language function.

\section{Discussion}

This child had no evidence of an underlying metabolic cause for his hyperammonaemic encephalopathy. With normal glucose, liver function tests, organic acids, amino acids, and orotic acid profile there was no evidence for Reye's syndrome, a urea cycle defect or organic acidaemia. 
We believe that, as a complication of cystoscopy, he developed a urinary tract infection with a urea splitting organism leading to raised urine ammonia. We did not isolate such an organism but this could have been because of suppression by antibiotics and high urine flow following drainage. ${ }^{1}$ Certain urea splitting organisms such as Ureaplasma urealyticum and Corynbacterium urealyticum are difficult to culture. ${ }^{2}$ In solution, ammonia exists in equilibrium with the ammonium ion. At $\mathrm{pH} 7.4$ about 1 in 100 molecules exist as ammonia whereas at $\mathrm{pH} 9$ the proportion rises to 1 in 2 . Ammonia is lipid soluble and rapidly crosses cell membranes of a dilated tract. ${ }^{3}$ The raised $\mathrm{pH}$ of the urine $(\mathrm{pH} 9)$ relative to that of venous blood favoured non-ionic diffusion of ammonia directly into the inferior vena cava via internal iliac veins. The ammonia bypassed the portal circulation avoiding hepatic detoxification. Hyperammonaemia led to glutamine mediated toxicity, astrocytic swelling, cerebral oedema, and raised intracranial pressure leading to transtentorial herniation.

Hyperammonaemic encephalopathy from infection with a urea splitting organism, usually Proteus mirabilis, in a dilated urinary tract has been reported previously, with a similar pathophysiological mechanism described. In contrast to our patient all the previous cases, seven children and one adult, had a definite anatomical abnormality. Six had prune belly syndrome, ${ }^{134}$ one an obstructive ureterocele, ${ }^{5}$ and one a neurogenic bladder. ${ }^{6}$ All made a full recovery. The peak concentrations of plasma ammonia were higher than in our case in two reports. ${ }^{16}$ It may be that the poor outcome in our patient was caused by a more prolonged exposure to raised ammonia.

This case emphasises the importance of recognising mood change, irritability, and alteration in consciousness levels as possible signs of developing hyperammonaemic encephalopathy. Severe hyperammonaemic brain injury is a rare but potentially preventable sequel to infection in a dilated urinary tract.

1 Ullman MA, Haecker TA, Medani CR. Hyperammonemic encephalopathy and urinary obstruction. $N$ Engl $7 \mathrm{Med}$ encephalopathy

2 Rose GA, Rosenbaum TP. Recurrent infection stones with apparently negative cultures. The case for blind antibacterial treatment. Br f Urol 1992;69:234-9.

3 Samtoy B, DeBeukalaer MM. Ammonia encephalopathy secondary to urinary tract infection with proteus mirabilis. Pediatrics 1980;65:294-7.

4 Das A, Henderson D. Hyperammonemic encephalopathy in a four year old child with prune belly syndrome. Pediatr Inf Dis f 1996;15:923, 925.

5 Sinha B, Gonsalez R. Hyperammonemia in a boy with obstructive ureterocele and proteus infection. $\mathcal{F}$ Urol 1984; 131:330-1.

6 Drayna JC, Titcomb CP, Varma RR, Soergel KH. Hyperammonemic encephalopathy caused by infection in a Hyperammonemic encephalopathy caused by infection
neurogenic bladder. $N$ Engl 7 Med 1981;304:766-8. 